[Case Report]

\title{
Synchronous Occurrence of Ovarian Seromucinous Carcinoma and Endometrial Mucinous Carcinoma: A Case Report
}

\author{
Ruka Urakawa ${ }^{1,5}$, Hiroshi Harada ${ }^{1 *}$, Atsushi Tohyama ${ }^{1}$, Yoko Aoyama ${ }^{1}$, Yasuyuki Kinjo $^{1}$, Kaori Hoshino $^{1}$, \\ Taeko UedA ${ }^{1}$, Seiji Kagami ${ }^{1}$, Jun Tsukamoto ${ }^{2}$, Takatoshi Aoki ${ }^{2}$, Ryuji Iwamura ${ }^{3}$, Masanori HisaokA ${ }^{3}$, \\ Yusuke MatsuURA $^{4}$ and Kiyoshi Yoshino ${ }^{1}$
}

${ }^{1}$ Department of Obstetrics and Gynecology, School of Medicine, University of Occupational and Environmental Health, Japan. Yahatanishi-ku, Kitakyushu 807-8555, Japan

2 Department of Radiology, School of Medicine, University of Occupational and Environmental Health, Japan. Yahatanishi-ku, Kitakyushu 807-8555, Japan

${ }^{3} 1$ st Department of Pathology and Oncology, School of Medicine, University of Occupational and Environmental Health, Japan. Yahatanishi-ku, Kitakyushu 807-8555, Japan

${ }^{4}$ Department of Nursing of Human Broad Development, School of Health Sciences, University of Occupational and Environmental Health, Japan. Yahatanishi-ku, Kitakyushu 807-8555, Japan

${ }^{5}$ Department of Obstetrics and Gynecology, Kyushu Rosai Hospital, Japan Organization of Occupational Health and Safety. Kokuraminami-ku, Kitakyushu 800-0296, Japan

\begin{abstract}
Endometrioid carcinoma is the most common histological type of concurrent synchronous cancers of the uterus and ovary. Here we report a case of synchronous seromucinous carcinoma of the ovary and mucinous carcinoma of the endometrium with a literature review. A 51-year-old multiparous female complained of irregular bleeding and shortness of breath. Computed tomography revealed a large pelvic mass that consisted of cystic and solid components, a tumor of the endometrium, and a large amount of pleural effusion. An endometrial biopsy indicated adenocarcinoma, and adenocarcinoma cells were found in the pleural fluid. The patient with advanced ovarian cancer or endometrial cancer with massive pleural effusion received three courses of neoadjuvant chemotherapy (NAC) with paclitaxel and carboplatin followed by interval debulking surgery (IDS). The NAC was effective, and IDS was performed with no gross residual lesions. The post-operative diagnosis was seromucinous carcinoma of the ovary in FIGO (2014) stage IVA (ypT3cNxM1a) and mucinous carcinoma of the endometrium in FIGO (2008) stage IA (ypT1aNXM0). Three courses of postoperative TC therapy were performed, and maintenance therapy with Bevacizumab is ongoing. The patient is well without evidence of recurrence, sixteen months after surgery.
\end{abstract}

Keywords : ovarian cancer, endometrial cancer, synchronous carcinoma, seromucinous carcinoma, mucinous carcinoma.

(Received January 12, 2021, accepted February 23, 2021)

\footnotetext{
*Corresponding Author: Hiroshi HARADA, Department of Obstetrics and Gynecology, School of Medicine, University of Occupational and Environmental Health, Japan. 1-1, Iseigaoka, Yahatanishi-ku, Kitakyushu 807-8555, Japan. Tel: +81-93-691-7449, Fax: +81-93-691-9337, E-mail: suigetsuisya@med.uoeh-u.ac.jp
} 


\section{Introduction}

The frequency of multiple cancers in female genital malignancies is reported to be $1-2 \%$ [1]. Endometrioid carcinoma is the most common histological type of multiple cancers of the uterus and ovary, and the absence of an endometrioid carcinomatous component is rare. Among those multiple cancers, advanced cancer is less common, and the prognosis is better than that of a sole advanced-stage cancer of uterine or ovarian origin. Seromucious carcinoma originating from the ovary is also relatively rare, and there have been no reports of distant metastasis [2-4]. Here we report a rare case of simultaneous multiple cancers with seromucinous carcinoma of the ovary and mucinous carcinoma of the uterus, with a literature review.

\section{Case History}

The patient was 51 years old, 2 gravida and 2 para. Her menstruation was of the 28-day type. She had a history of hypertension, and, in her family history, her father had lung cancer. She started having irregular vaginal bleeding around the age of 50, and she visited an obstetrician's clinic, where cervical and endometrial cytology and transvaginal ultrasonography were performed, but no obvious abnormality was found. She continued to have intermittent irregular bleeding over the following months. Shortness of breath appeared about 10 months after her first examination, and she was examined by a local physician. A chest X-ray showed a right pleural effusion, and the patient was referred to the Department of Pulmonology at our hospital. A pleural effusion cytology revealed adenocarcinoma cells, and a CT scan showed a left adnexal mass with solid growth, an intrauterine mass, perito-
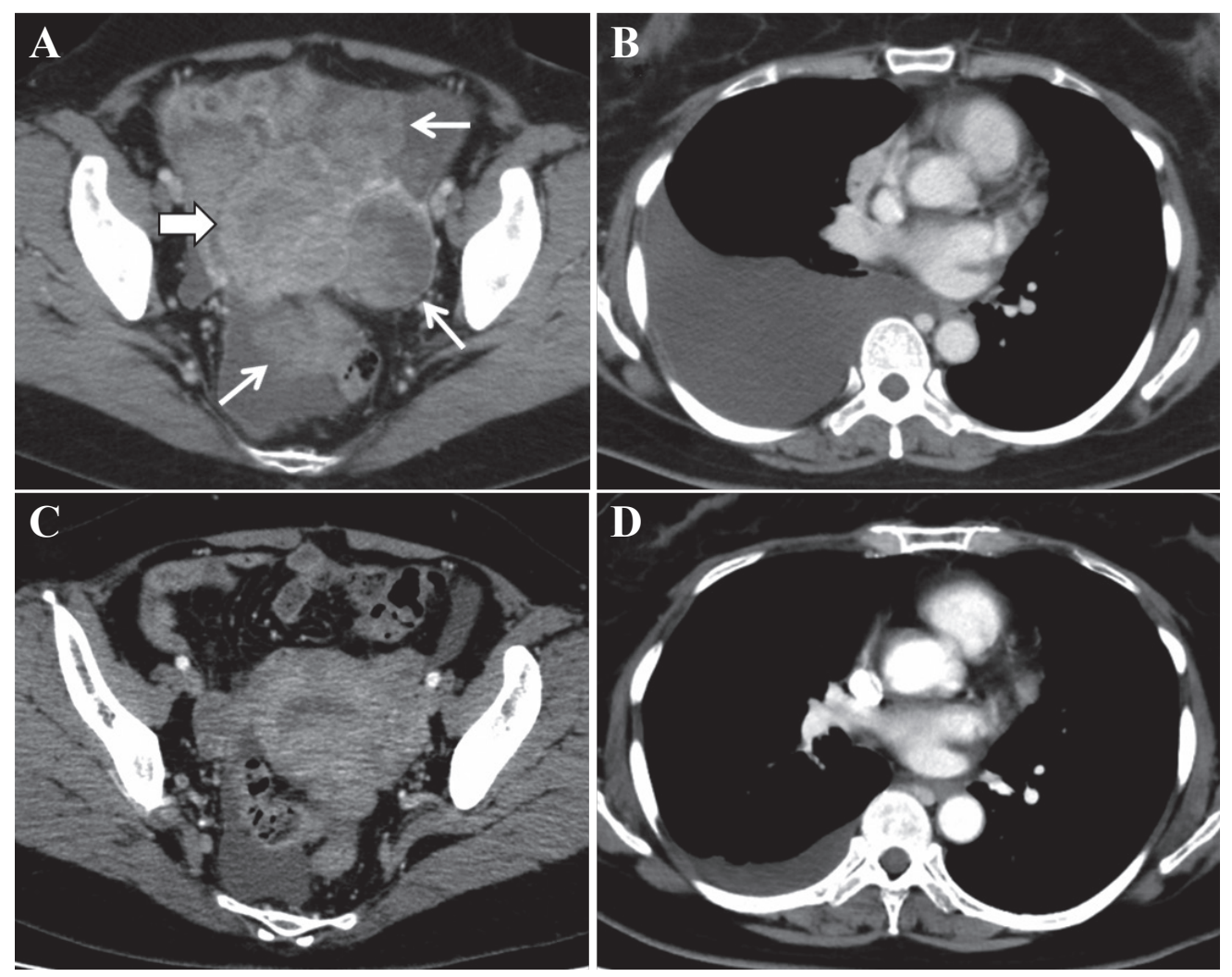

Figure 1. CT scan images. A: Pre-treatment contrast-enhanced CT images show a left adnexal mass with solid growth $(\Rightarrow)$, disseminated masses $(\Rightarrow)$, and B: a right pleural effusion. C, D: Contrastenhanced CT images after NAC showed a reduction in the adnexal mass and a decrease in pleural effusion. $\Rightarrow$ : adnexal mass, $\Rightarrow$ : uterine mass, NAC: neoadjuvant chemotherapy. 
neal dissemination, ascites, and a right pleural effusion (Figure 1A, B). She consulted our department with suspicion of advanced ovarian or uterine cancer.

On initial examination, transvaginal ultrasonography revealed a $13 \mathrm{~cm}$ solid and cystic mass on the left side of the pelvis. The endometrium was $16 \mathrm{~mm}$ thick. A pelvic examination revealed that the uterus was the size of a hen's egg with poor mobility, and the bilateral adnexa were difficult to identify. Tumor markers of CA19-9 and CA125 were elevated: the CA19-9 was $1,415 \mathrm{U} / \mathrm{m} l(<37 \mathrm{U} / \mathrm{m} l$ reference value $)$ and the CA125 was $9,564 \mathrm{U} / \mathrm{m} l(<35 \mathrm{U} / \mathrm{m} l$ reference value). A pelvic MRI scan revealed multiple masses in and around the uterus. A T1-weighted MR image after contrast administration indicated hypovascularity and myometrial invasion of the intrauterine mass. A $13 \mathrm{~cm}$ hyperintense papillary mass and a $5 \mathrm{~cm}$ solid and cystic mass on the left side of the uterus were found in T2-weighted images (Figure 2A, B). A PET-CT scan showed hyperaccumulation of the uterus and left ovary in a cluster (SUVmax 20.68). There was no abnormal accumulation suggestive of lymph node metastasis. Pleural effusion cytology revealed the presence of adenocarcinoma (Figure 3A), while endometrial cytology and histopathology revealed adenocarcinoma with the characteristics of mucinous carcinoma.

Based on the above pre-treatment examinations, advanced ovarian cancer or uterine cancer was suspected, and the possibility of double cancer was also considered. Three courses of paclitaxel plus carboplatin (TC) therapy were administered as preoperative neoadjuvant chemotherapy. After 3 courses of TC therapy, the reduction rate of the uterine mass was $37 \%$ and that of the left ovarian tumor was $69 \%$. An overall reduction rate of $64 \%$ was found to be a partial response (PR) (Figure 1C, D), and the interval debulking surgery (IDS) strategy was adopted. In the laparotomy findings of IDS, the uterus and right adnexa were normal in size and appearance. An $8 \mathrm{~cm}$ left ovarian tumor was found highly adherent to the dorsal uterus, Douglas fossa, and retroperitoneum. Large amounts of ascites were present and ascitic cytology was positive (Figure 3B). Total hysterectomy, bilateral adnexal resection, and partial omentectomy were performed (intraoperative blood loss: $660 \mathrm{ml}$, operation time: 4 hours 6 minutes).

The tumors arising in the uterine corpus and the left ovary had different histopathological features. The tumor in the uterine corpus was found to be a well-differentiated mucinous carcinoma having PAS-positive intracytoplasmic mucin, mimicking the endocervical epithelium arranged in a papillary or tubular growth fashion with a minor endometrioid carcinomatous component (10\%), invading down to the upper portion of the myometrium $(4 / 18 \mathrm{~mm})$. No venous invasion or lymphatic permeation was identified. Immunohistochemically, the tumor cells were positive for ER and CK7, and negative for p53 and CK20 (Figure 4 A-G).
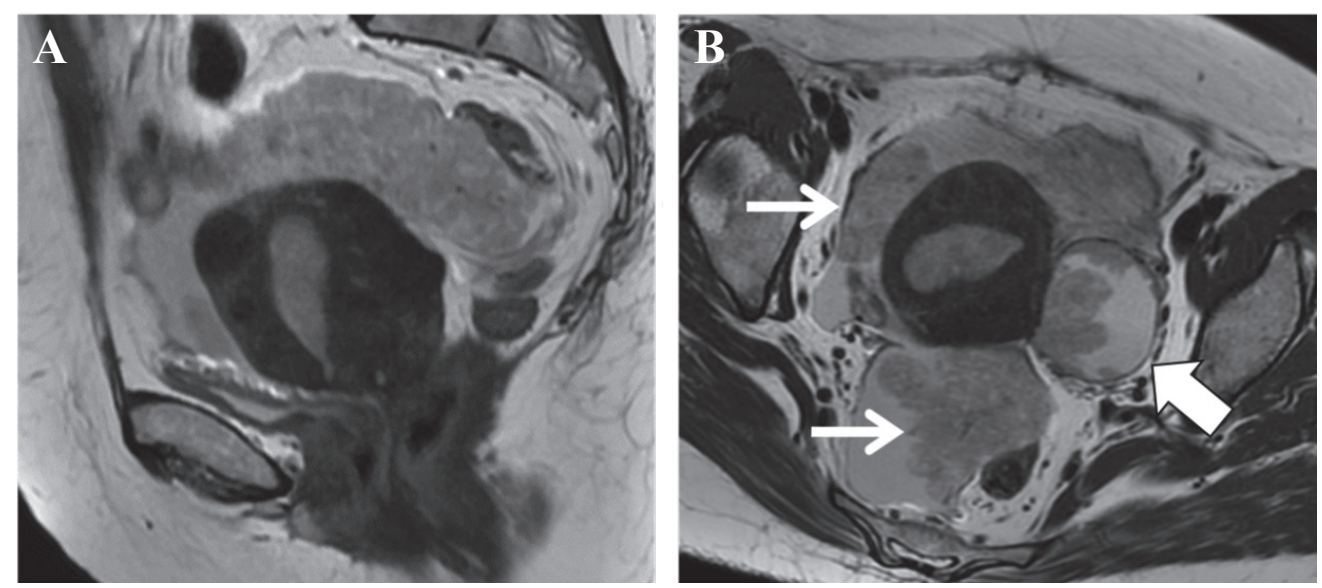

Figure 2. MRI scan images. A, B: T2-weighted images show a low-signal mass in the uterus, and cross-sectional images show a cystic mass $(\Rightarrow)$ in the adnexal region with internal solid parts on the left side of the uterus and a peritoneal disseminated lesion $(\Rightarrow)$. 

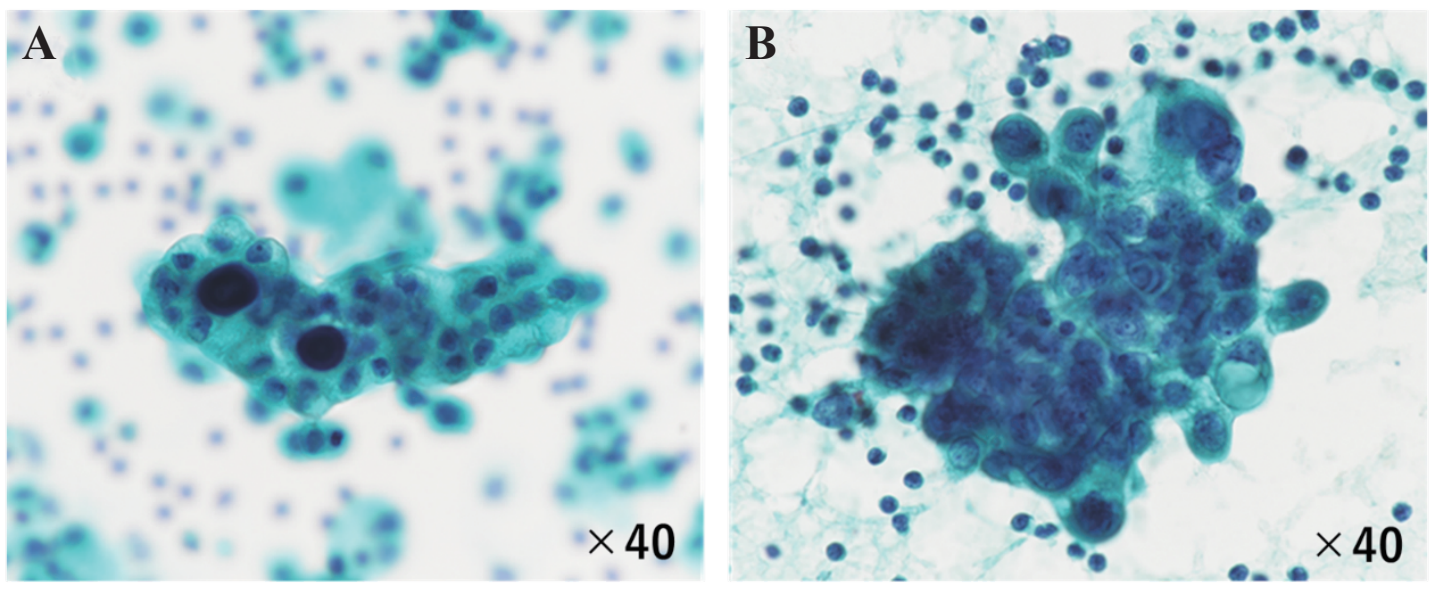

Figure 3. Cytological examination (Papanicolaou staining). A: Pleural fluid cytology and B: ascites fluid cytology. In pleural and ascitic fluid, atypical cells with enlarged nuclei and increased chromatin appear. The small round shape of the cells with a high $\mathrm{N} / \mathrm{C}$ ratio and, in some cases, a relatively rich cytoplasm. The cytological features are similar to those in histological specimens of seromucinous carcinoma of the ovary. N/C ratio: nuclear/cytoplasmic ratio.

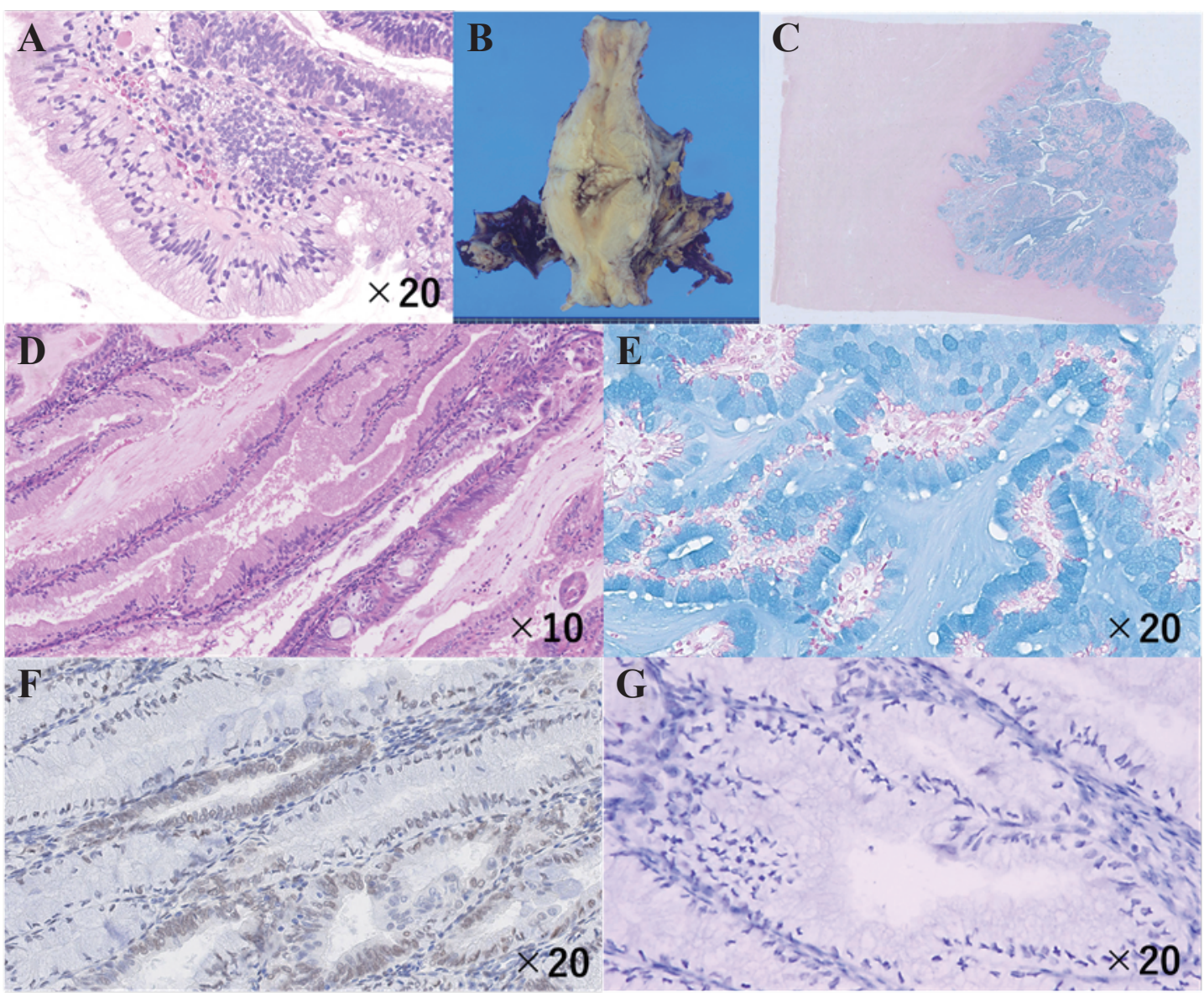

Figure 4. Uterus Histopathology. A: H\&E staining (biopsy), B: Macro image, C: Loupe image, D: H\&E staining, E: Alcian blue staining, F: ER staining, G: p53 staining. The histopathology of the uterine tumor shows a well-differentiated adenocarcinoma with abundant mucus. External growth patterns were seen with little evidence of muscular invasion. Those atypical cells were positive for Alcian blue staining and negative for p53 immunostaining. 
The left ovarian tumor had a solid growth macroscopically. The tumor was composed of a proliferation of atypical cells arranged in a papillary or cribriform growth resembling endocervical glands and more highly atypical cells forming papillary structures, corresponding to seromucinous carcinoma combined with serous carcinoma components. Those atypical cells also formed cribriform or fused glandular ducts and showed infiltration, and some parts of the tumor had atypical endometrioid columnar or squamoid cells. Alcian blue staining of the left ovarian tumor was poorly stained and clearly different from that of the uterine corpus tumor (Figure 5A-H). Immunostaining showed that the ovarian atypical cells were positive for ER, CK7, p53, and CEA, and negative for WT-1, CK20, and MUC2.

The tumor in the uterine corpus showed only superficial muscular infiltration on pretreatment imaging and examination of the excised specimen, and there was continuity between the left ovarian tumor and peritoneal disseminated lesions in intraoperative findings. Considering the similarity in the pleural and ascitic fluid cytology, we confirmed the diagnosis of multiple cancers for the left ovarian cancer stage IVA (ypT3cNxM1a, seromucinous carcinoma) and uterine cancer stage IA (ypT1aNxM0, mucinous carcinoma). The germline mutation of the BRCA1/2 gene was negative. After IDS, 3 courses of TC therapy (total 6 courses) were administered and bevacizumab was administered after the 6 th course. Bevacizumab was continued as a single agent, and the patient was free of recurrence at 16 months postoperatively.

\section{Discussion}

Both seromucinous carcinoma of the ovary and mucinous carcinoma of the uterine corpus have recently been recognized as pathological subtypes of endometrioid carcinoma. There have been no reports to date of a combination of these two types in the concurrent synchronous cancers of the uterus and ovary, and this case is the first report of such a combination. Only one case of multiple tumors, a mucinous carcinoma of the uterine corpus and a benign bilateral ovarian mucinous tumor found by chance during infertility treatment, has been reported in Japan [5].
This case was diagnosed as simultaneous multiple cancers based on the Ulbright and Ree diagnostic criteria $[6,7]$. Multiple cancers in female genital malignancies are relatively rare, occurring in approximately $1-2 \%$ of all cases, and the most frequent multiple cancer patterns are endometrial and ovarian cancers, which are reported to account for approximately $45 \%$ of them [1-4]. Those multiple cancers have a peak incidence in the late 40 s and are more common in perimenopausal women. This feature is consistent with the histological tendency to have more endometrioid carcinomas $[3,8]$.

In this case, mucinous carcinoma was found in the uterine corpus before starting treatment. The frequency of primary mucinous carcinoma in the uterine corpus ranges from $1-9 \%$ of all uterine cancers. Mucinous carcinoma of the endocervical type is classified histologically as a subtype of endometrioid carcinoma, and the treatment is equivalent to that of endometrioid carcinoma. The majority of cases are detected at stage I, and the prognosis is favorable $[9,10]$.

Seromucinous carcinoma of the ovary belongs to a new category called "seromucinous tumors", which was defined by the WHO classification in 2014, although its exact incidence and prognosis are unknown due to the small number of reported cases. Histopathologically, seromucinous carcinoma shows papillary projections and an admixture of multiple Müllerian duct-derived cells, similar to serous borderline tumors, with high-grade cytological atypia and structural complexity. A cribriform and solid growth pattern is commonly observed, and the pattern of stromal invasion is divided into exclusion and destruction, such as in mucinous carcinoma. The mitotic image is not high and is often less than 5/10 high-power fields (HPF) [11].

The immune profile of seromucinous tumors is characterized by a high frequency of ER and PR expression, low frequency of WT-1 expression, and lack of CK20 and CDX2 expression, which is also consistent with the immunostaining pattern of Müllerian duct-derived tumors. Seromucinous tumors are also highly associated with endometriosis and are similar to endometriosis-associated tumors in terms of genetic variants. ARID1A mutations/lack of expression of ARID1A have been reported in approximately $50 \%$ of endometriosis and clear cell carcinomas; and ARID1A 

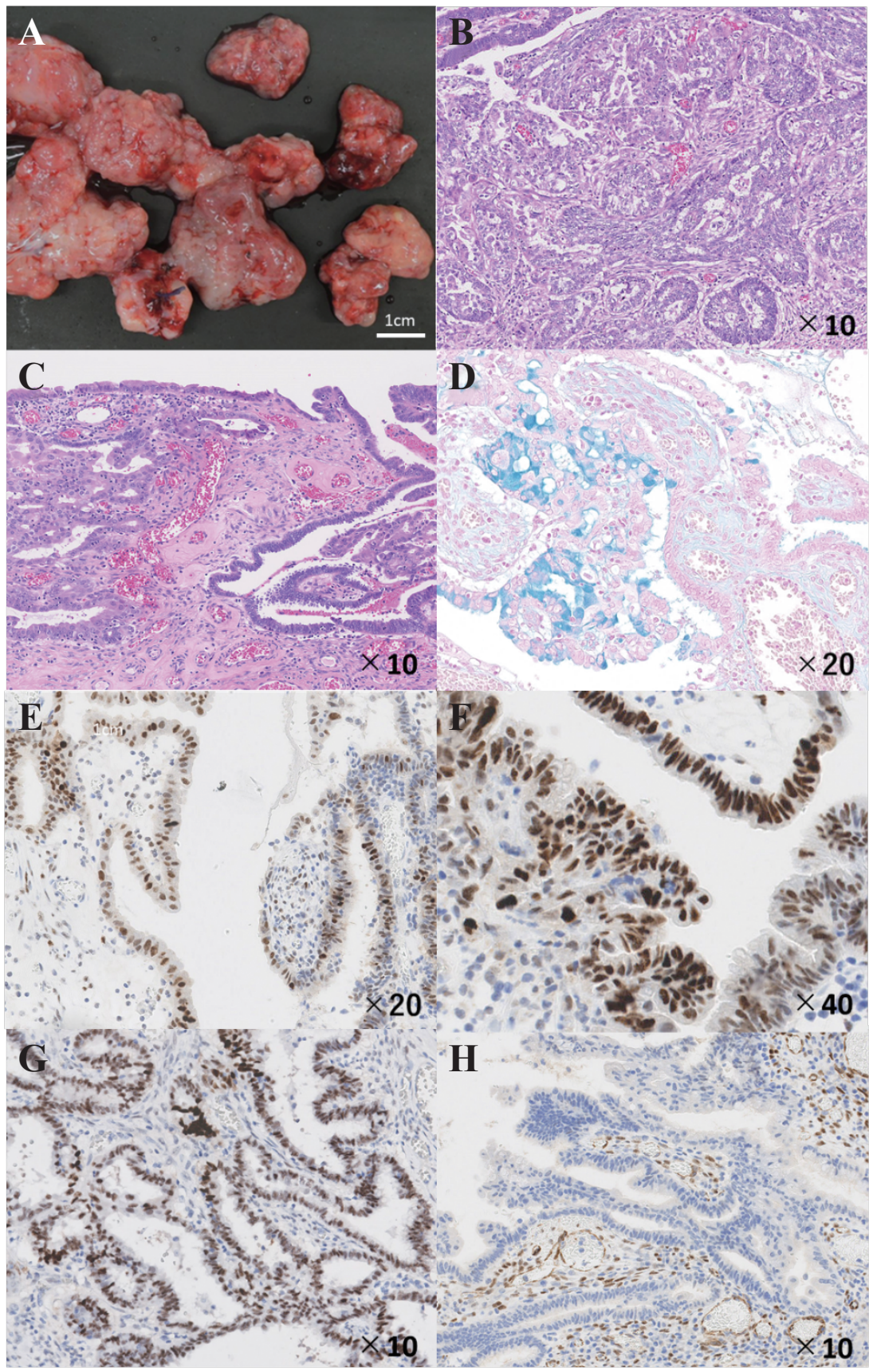

Figure 5. Histopathological image. A: Macro image, B, C: H\&E staining, D: Alcian blue staining, E, F: p53 staining, G: ER staining, H: WT-1 staining. The macro-image of the ovarian tumor showed fine papillary structures in the superficial layer of the tumor. Histological findings showed the coexistence of a cytoplasmic-poor atypical cell population similar to serous carcinoma and a round- or oval-shaped atypical cell population with a small amount of mucus. The round tumor cells had an Alcian blue-positive mucus, and immunostaining was positive for p53 and ER, and negative for WT-1.

mutations/lack of expression of ARID1A have recently been noted in seromucinous tumors as well. This is in contrast to serous and intestinal mucous tumors, which often do not show mutations in ARID1A. There is also a report of 32 cases morphologically diagnosed as seromucinous carcinoma of the ovary, which were analyzed in detail by immunohistological approach and next-generation sequencing. KRAS (70\%), PIK3CA 
(37\%), PTEN (19\%) and ARID1A (16\%) mutations were identified in the gene mutation profile, while, in contrast, no CTNNB1 mutations were identified. After integrating immunohistochemistry and mutation profiles, most seromucinous ovarian cancers were reclassified to endometrial carcinoma 23/32 (72\%), followed by low-grade serous carcinoma $8 / 32(25 \%)$ and mucinous carcinoma $1 / 32(3 \%)$, according to one report [12].

On the basis of clinicopathological or immunohistochemical and molecular genetic characteristics, some reports suggest that a more appropriate designation for seromucinous tumors is "mixed Müllerian ductal tumors" $[13,14]$. Thus, the category of seromucinous carcinoma is also considered as a subtype of endometrioid carcinoma, and there is some variation in the disease units.

Paclitaxel plus carboplatin (TC) therapy is one of the typical standard treatments for both uterine and ovarian cancers. In the case of our patient, adjuvant chemotherapy was administered due to the possibility of microscopic dissemination. Seromucinous carcinoma of the ovary is less common, and the efficacy of chemotherapy or additional postoperative treatment is unknown. There are no reports of the co-existence of seromucinous carcinoma of the ovary with mucinous carcinoma of the uterine corpus [15]. The prognosis of multiple gynecological cancers has been reported to be relatively good, since they are often distinct earlystage cancers, and the 5 -year survival rate has been reported to be $85.9 \%$ [16-18]. However, only a few of these reports include advanced ovarian cancer. While a study of early-stage ovarian cancer cases limited to mucinous or seromucinous histology indicated that surgical treatment may affect prognosis, there are no reports that refer to a standard treatment for advanced seromucinous carcinoma [12].

\section{Conclusions}

As stated above, seromucinous carcinoma is considered to be a subtype of endometrioid carcinoma, and the standard treatment for endometrioid carcinoma was followed in the present case. Although there is a lack of information on a standard treatment for multiple cancers with a rare histological pattern, our ex- perience with this case suggests that TC therapy with bevacizumab, followed by bevacizumab alone, may have been beneficial in prolonging progression-free survival.

\section{Conflict of Interest}

The authors of this article have no conflict of interest.

\section{References}

1. Singh N (2010): Synchronous tumours of the female genital tract. Histopathology 56(3): 277-285

2. Eisner RF, Nieberg RK \& Berek JS (1989): Synchronous primary neoplasms of the female reproductive tract. Gynecol Oncol 33(3): 335-339

3. Gilks CB \& Kommoss F (2018): Synchronous tumours of the female reproductive tract. Pathology 50(2): 214-221

4. Sozen H, Vatansever D, Iyibozkurt AC et al (2015): Clinicopathologic and survival analyses of synchronous primary endometrial and epithelial ovarian cancers. J Obstet Gynaecol Res 41(11): 1813-1819

5. Gotoh T, Hayashi N, Takeda S, Itoyama S, Takano M \& Kikuchi Y (2004): Synchronous mucinous adenocarcinoma of the endometrium and mucinous cystadenoma of bilateral ovaries presenting during fertility therapy. Int J Gynecol Cancer 14(1): 169-171

6. Ulbright TM \& Roth LM (1985): Metastatic and independent cancers of the endometrium and ovary: a clinicopathologic study of 34 cases. Hum Pathol 16(1): 28-34

7. Ree YS, Cho SH, Kim SR, Cho SH, Kim KT \& Park MH (2003): Synchronous primary endometrial and ovarian cancer with three different histologic patterns: A case report. Int J Gynecol Cancer 13(5): 678-682

8. Taylor J \& McCluggage WG (2015): Ovarian seromucinous carcinoma: report of a series of a newly categorized and uncommon neoplasm. Am J Surg Pathol 39(7): 983-992

9. Ross JC, Eifel PJ, Cox RS, Kempson RL \& Hendrickson MR (1983): Primary mucinous adenocarcinoma of the endometrium. A clinicopathologic and histochemical study. Am J Surg Pathol 7(8): 715-729

10. Melhem MF \& Tobon H (1987): Mucinous adenocar- 
cinoma of the endometrium: a clinico-pathological review of 18 cases. Int J Gynecol Pathol 6(4): 347-355

11. Nagamine M \& Mikami Y (2020): Ovarian Seromucinous Tumors: Pathogenesis, Morphologic Spectrum, and Clinical Issues. Diagnostics (Basel) 10(2): 77

12. Rambau PF, McIntyre JB, Taylor J et al (2017): Morphologic Reproducibility, Genotyping, and Immunohistochemical Profiling Do Not Support a Category of Seromucinous Carcinoma of the Ovary. Am J Surg Pathol 41(5): 685-695

13. Shappell HW, Riopel MA, Smith Sehdev AE, Ronnett BM \& Kurman RJ (2002): Diagnostic criteria and behavior of ovarian seromucinous (endocervical-type mucinous and mixed cell-type) tumors: atypical proliferative (borderline) tumors, intraepithelial, microinvasive, and invasive carcinomas. Am J Surg Pathol 26(12): 1529-1541

14. Kurman RJ \& Shih Ie M (2016): Seromucinous Tumors of the Ovary. What's in a Name? Int J Gynecol Pathol
35(1): 78-81

15. Ledermann JA, Luvero D, Shafer A et al (2014): Gynecologic Cancer InterGroup (GCIG) consensus review for mucinous ovarian carcinoma. Int J Gynecol Cancer 24(9 Suppl 3): S14-S19

16. Castro IM, Connell PP, Waggoner S, Rotmensch J \& Mundt AJ (2000): Synchronous ovarian and endometrial malignancies. Am J Clin Oncol 23(5): 521-525

17. Musa F, Huang M, Adams B, Pirog E \& Holcomb K (2012): Mucinous histology is a risk factor for nodal metastases in endometrial cancer. Gynecol Oncol 125(3): 541-545

18. Soliman PT, Slomovitz BM, Broaddus RR et al (2004): Synchronous primary cancers of the endometrium and ovary: a single institution review of 84 cases. Gynecol Oncol 94(2): 456-462

J UOEH $43(2): 235-242(2021)$ 